\title{
The Phenomena of Spin in Detonation
}

$\mathrm{C}^{\mathrm{A}}$ AMPBELL and Woodhead ${ }^{1}$ discovered that in certain gaseous mixtures, when a detonating type of combustion flashed along a cylindrical tube, the flame front was not simply a disc-like or convex surface, but the detonation spun spiralwise along the tube, giving rise to a banded appearance in the photographs of the luminous products of combustion. Bone and Fraser ${ }^{2}$ made a careful photographic investigation of the phenomenon, which showed that the initiation of detonation was almost

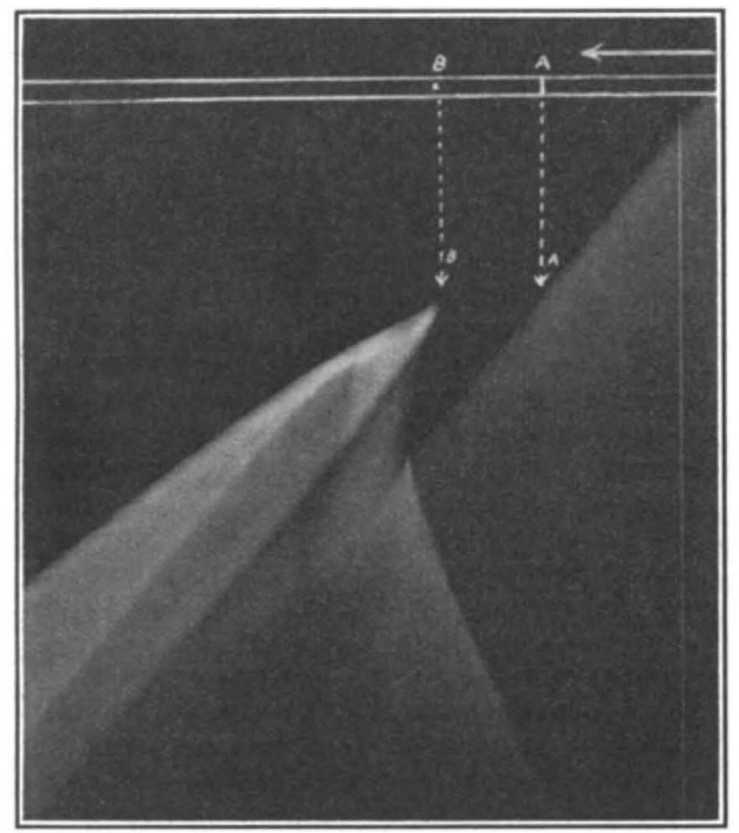

FIr. 1.

invariably associated with the spin of the 'head' of detonation but that in hydrogen mixtures it rapidly disappeared, whereas in certain mixtures it was visible in the ordinary flame before the detonating condition had been reached. They came to the conclusion that the frequency of the spin was fairly independent of the composition of the mixture, of its density and of the speed with which the detonation passed down the tube, but that it depended on the tube diameter such that the pitch of the rotation of the 'head' was about three times the tube diameter. Those results were obtained using a 9 in. duralumin drum rotating at 16,000 r.p.m. (equivalent to a film speed of $190 \mathrm{~m}$./sec.). The extension of that investigation ${ }^{3}$, which will be briefly described in this article, has been rendered possible by the use of a special camera whereby photographs could be taken at speeds which would be equivalent to using a film rotating on a drum at 1,000 metres a second, thus enabling events to be recorded which happen during a millionth of a second.

The apparatus consists essentially of a doublesided stainless steel mirror which is rotated at high speed, and flashes the image of the explosion formed by a suitable lens (Dallmeyer Pentac 12 in. focus) on to the surface of a circularly disposed stationary photographic film. In order to rotate the mirror at the high speed (30,000 r.p.m.), which is equivalent to a speed of rotation of the image of 1,000 per sec., the mounting has to be such that a vacuum can be maintained within the camera. The drive is by means of a 4 h.p. motor which can be maintained at any constant speed up to 6,000 r.p.m. by an auxiliary dynamo, the voltage output of which is controlled by altering the resistance in the shunt-field circuit; this, together with the friction drive off a spherical steel surface which is rotated by the motor, enables the most suitable speed to be chosen so as to provide the most accurate measurement of the flame. The explosive mixture is usually fired by a spark and travels down a 7 metre length of copper tube and then through a special tap into a glass tube $1.3 \mathrm{~cm}$. diameter and $1 \frac{1}{2}$ metres long which is placed in the field of view of the camera lens. Most of the experiments were made with $2 \mathrm{CO}+\mathrm{O}_{2}$ mixtures.

It is only possible here to mention a few of the results of the investigation; the photographs provide detail of the phenomena of detonation never before attained. It has often been noticed ${ }^{4}$, when the detonating type of combustion is initiated, that where this initiation occurs is somewhat ahead of the advancing flame front. The present investigation shows quite definitely that this is generally the case. The composite character of the setting up of the detonation is clearly shown in Fig. 1. The flame travels forward at 1,275 m./sec. ; it so influences the combustible molecules in a compression wave ahead that ignition suddenly occurs at $B, 6 \cdot 3 \mathrm{~cm}$. from the flame, which has reached position $A$, and from the position $B$ a detonation wave travels forward at $3,260 \mathrm{~m} . / \mathrm{sec}$. The velocity of this wave soon subsides, however, to $1,980 \mathrm{~m} . / \mathrm{sec}$. A wave also starts in the backward direction from the seat of detonation through the gases with a resultant forward velocity of $350 \mathrm{~m}$./sec., and gives rise on meeting the advancing flame to the 'retonation' wave travelling back through the products at $875 \mathrm{~m}$./ $/ \mathrm{sec}$. Not only 


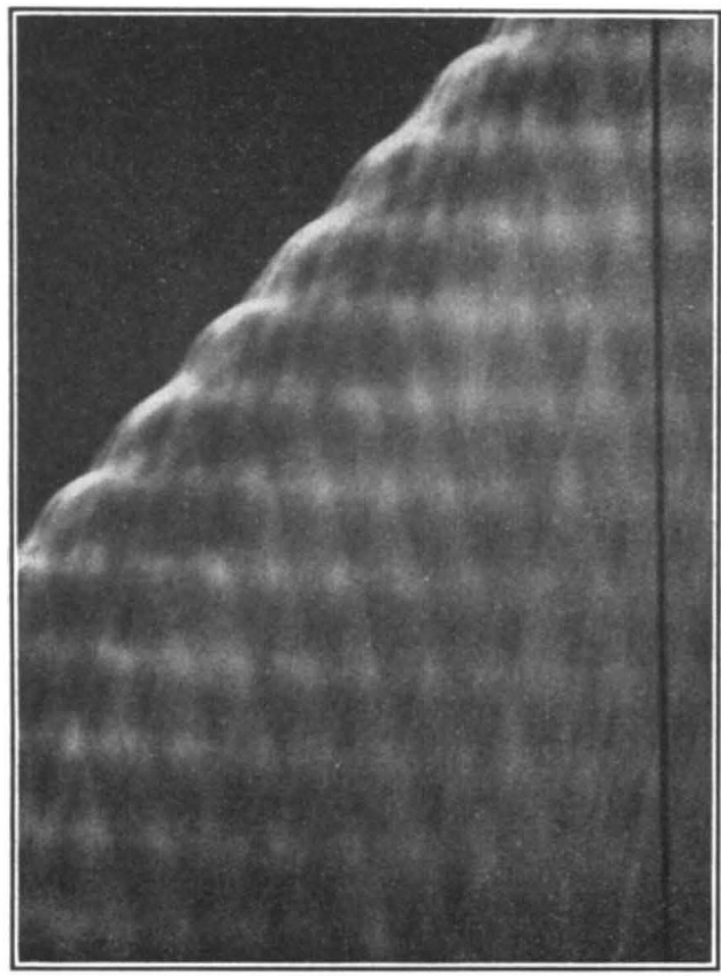

Fro. 2.

is a compression wave visible which travels out from the point of auto-ignition at $1,320 \mathrm{~m} . / \mathrm{sec}$., but also, at the point where the initial rapid detonation in the forward direction subsides to the more normal velocity, a compression wave is visible which moves forward, also at $1,320 \mathrm{~m} . / \mathrm{sec}$. The spin first makes its appearance at this point. The whole interval from the point when autoignition occurs to the point when the velocity subsides to $1,980 \mathrm{~m} . / \mathrm{sec}$. is only about a ten thousandth of a second.

The spin is due to the spiral motion of the head of detonation down the tube, and the pitch appears to be governed mainly by the diameter of the tube, as was shown by causing a detonation to proceed down a tapered tube. It is possible to observe the phenomenon of spin and to maintain detonation in $2 \mathrm{CO}+\mathrm{O}_{2}$ mixtures in tubes so small as $3 \cdot 6 \mathrm{~mm}$. diameter. In tubes of triangular or square section the pitch of the spin is nearly that which would occur in a tube of circular section of equal periphery; in a tube of oblong section the track is that in a tube of circular section the periphery of which corresponds to the shorter sides of the oblong.

The banded appearance of the after-flame which was obtained in former investigations is now shown by the higher resolution to be due to the criss-crossing of a set of waves or regions of high luminosity starting out from the spinning head twice during each rotation and proceeding at an angle of $22^{\circ}$ to each other, one in the forward direction and the other slightly retrogressive (see Fig. 2).

The authors state that "whether or not a persistent spin develops in a detonation seems to depend on the requisite degree of excitation of the combustible gas. In cases (such as when hydrogen is involved) where this is easily attained, the initial close association between the forward moving compression wave and the burning medium in the front does not become attenuated and no persistent spin can be developed. On the other hand, a persistent spin develops whenever the requisite degree of excitation of the molecules of the combustible gas is such as to render the association between the forward moving front precarious so that an attenuated (cone-shaped) front develops". They also state that "it follows geometrically that at each half-rotation of the head a pocket of the unburnt gas will be enclosed on

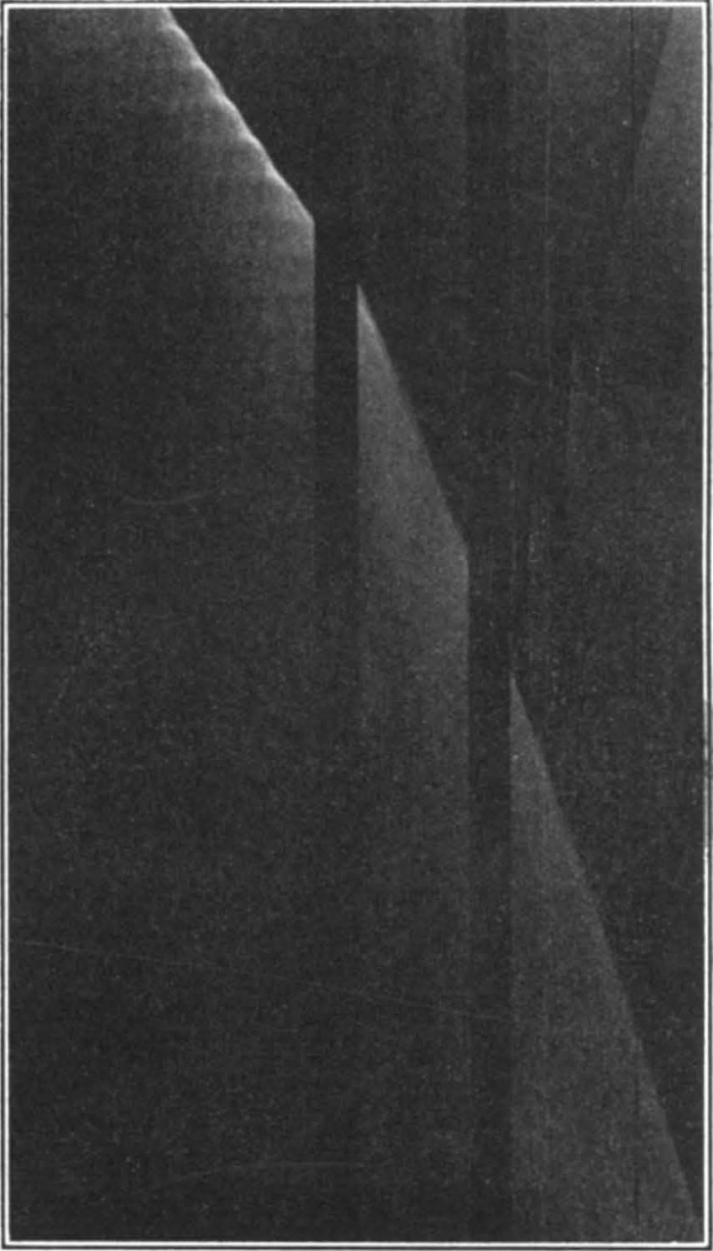

Fra. 3. 
three sides by the flame and thereby spontaneously ignited by its radiation", giving rise to the effects observed which accompany the spin.

The view that activated combustion must be associated with the compression wave in order to maintain detonation is supported by experiments in which a small $\frac{1}{4}$ in. nitrogen gap (obtained by means of the special tap) in the column of explosive gas causes the detonation and spin to be destroyed and only reinitiated when the flame front again catches up the compression wave. It is interesting to learn that, contrary to Dixon's results, the velocity and stability of the detonation wave is enhanced rather than diminished by intensively drying the carbon monoxide mixtures. Addition of hydrogen appears to give rise to a multiplicity of spinning heads, and more than 2 per cent of hydrogen tends to prevent the spin, though detonation is maintained.

The remainder of the present memoir relates to experiments on the effect of magnetic and electric fields on the propagation of detonation. Very strong magnetic fields were obtained by magnets of special design. A transverse field of 35,000 gauss had no appreciable effect on the detonation owing to the short time $(0.005 \mathrm{sec}$.) the flame is within the field, but longitudinal fields of 22,000 gauss over a length of $58 \mathrm{~cm}$., or of 35,000 gauss over $8 \mathrm{~cm}$., caused an appreciable slowing down of the rate of travel of the detonation by about $50 \mathrm{~m}$./ $\mathrm{sec}$. The effects of the magnetic field were not large. Strong electric fields (up to 5,000 volts per cm.), on the other hand, had a very marked effect, particularly when the flame travelled in the direction negative to positive potential; for example, when the detonation passed the negative electrode, both spin and detonation were suppressed, the flame speed decreasing to about $1,000 \mathrm{~m}$. $/ \mathrm{sec}$. by the time the positive electrode was reached and continuing to fall as it proceeded into the no-field region (see Fig. 3). For passage in the direction positive to negative there was a slight acceleration of the flame.

It is supposed that the positive $\mathrm{CO}^{+}$ions are important for the maintenance of activation in the detonation head, and that if these are drawn out of the flame front, the activation is not maintained and so the spin and eventually the detonation are destroyed by separating the region of activation from that of the region of high compression. This is borne out by the fact that in dry carbon monoxide mixtures or when hydrogen $(0 \cdot 3$ per cent) is present, the 'damping' influence of the field on the detonation is counteracted. In methane mixtures, too, the spin and velocity were not influenced by the field.

There can be no doubt that these experiments form a highly interesting addition to information about the behaviour of the detonating type of explosive in gaseous mixtures.

A. C. E.

\footnotetext{
${ }^{1}$ J. Chem. Soc., 3010 ; 1926.

${ }^{2}$ Phil. Trans., A, 230, 363; 1932.

sone, Fraser and Whecler, Phil. Trans., A, 235, 29; 1935.

Le Chatelier, C.R., 130, 1756; 1900. Egerton and Gates, Proc. Roy. Soc., A, 114, 137; 1927. Bone and Fraser, loc. cit.
}

\section{A Century of Botany* I835-I885-I935}

By Prof. F. O. Bower, F.R.S.

$\mathrm{U}^{\mathrm{r}}$ $P$ to 1885 , the whole field of botany was supposed to be covered by the professor himself. The elementary teaching might embrace the spirit of all its branches: but the science as a whole was then like a bomb with its pin drawn out, ready to burst into divergent lines for which neither personnel nor accommodation were prepared. Stimulating the time surely was, but exacting to the point of impossibility. The best course for the new professor in Glasgow was then to select some branch as his own special study, and by preference one cognate with local history. Personal experience gained elsewhere pointed to the mosses and ferns, a line of specialisation which

* Continued from $p .941$. would renew continuity with the Hookerian tradition. Moreover, the overcast skies of Glasgow gave conditions suitable for their culture. So after an interval of half a century, the special line of research followed there by Sir William Hooker was resumed.

With the new ordinances framed under the Act of 1889 came changes of organisation, which had the effect of levelling up the Scottish university system to that of England. The establishment of degrees in science stimulated higher courses in all the departments, and this led, of necessity, to their expansion both in staff and in equipment. Under the new ordinances, the differentiation of elementary and advanced classes involved an 